HCV-RNA, is not yet routinely available and is limited by false-positive or false-negative results.

Meanwhile, in order to reduce the incidence of $\mathrm{HCV}$ infection, strict adherence to the universal precautions (CDC, Atlanta) must be carried out. According to current knowledge the application of these measures of hygiene is probably sufficient to prevent HCV spread in the dialysis setting, and the isolation of positive patients is not warranted.

Department of Nephrology and

M. Beccari

Dialysis,

Fatebenefratelli and Oftalmico

Hospital,

Milan,

Italy

1. Sungur C. Is $\mathrm{HCV}$ a nosocomial infection in haemodialysis patients? Nephrol Dial Transplant 1994; 9(7): 875-876

2. McIntyre PG, McCruden EAB, Dow BC et al. Hepatitis C virus infection in renal dialysis patients in Glasgow. Nephrol Dial Transplant 1994; 9(3): 291-295

3. Jadoul M, Cornu C, van Ypersele de Strihou C, UCL Collaborative Group. Incidence and risk factors for hepatitis $C$ seroconversion in hemodialysis: a prospective study. Kidney Int 1993; 44: 1322-1326

4. Allander T, Medin C, Jacobson SH, Grillner L, Persson MAA. Hepatitis $\mathrm{C}$ transmission in a hemodialysis unit: molecular evidence for spread of virus among patients not sharing equipment. J Med Virol 1993; 43: 415-419

5. Caramelo C, Navas S, Alberola ML, Bermejillo T, Reyero A, Carreño V. Evidence against transmission of hepatitis $\mathrm{C}$ virus through hemodialysis ultrafiltrate and peritoneal fluid. Nephron 1994; 66: $470-473$

Sir,

I read Dr Beccari's contribution on the issue of nosocomial spread of hepatitis $\mathrm{C}$ virus (HCV) infection among haemodialysis patients with interest. He stresses another controversial aspect of the problem; the isolation policy for $\mathrm{HCV}$-infected patients in dialysis units, and concludes that if universal precautions are followed strictly, there is no need for such an isolation policy.

I agree with Dr Beccari that adherence to universal precautions is an essential measure in the prevention of nosocomial blood-borne viral infections, but on the other hand I think it is hard to draw a concrete conclusion about isolation policies for the following reasons:

1. Molecular biological studies which show the presence of viral genetic material in the haemodialysis ultrafiltrate rather than the ones which fail to detect the viral RNA should be regarded as important microbiological findings. Apart from methodological differences and errors, the degree of viraemia of a patient is probably an important factor in the contamination of the ultrafiltrate.

2. Haemodialysis treatment itself is a well-established risk factor for acquiring $\mathrm{HCV}$ infection [1], especially if the same machine is shared by more than four patients [2].

3. In the previous decade isolation policies for hepatitis $B$ virus (HBV) infection, which shares common routes of transmission with $\mathrm{HCV}$, has been successful in reducing the nosocomial transmission of HBV infection in dialysis units, and isolation policies for $\mathrm{HBV}$-infected dialysis patients are mandatory.

4. The risk of acquiring nosocomial $\mathrm{HCV}$ infection is strikingly different in units with a low rate of infection from those in which $50-70 \%$ of chronic dialysis patients are infected.
5. The issue becomes more complex when one remembers the lack of protective effect of anti-HCV antibodies, different infectivity and pathogenicity potentials of distinct $\mathrm{HCV}$ strains, and studies showing dialysis patients infected with more than one strain of $\operatorname{HCV}[3,4]$.

The answer to this controversy probably resides in the data which will be derived from the molecular epidemiolog ical studies. Demonstration of a particular strain of $\mathrm{HCV}$ in infected patients sharing the same machine and/or dialysis unit will be an invaluable additional finding in favour of nosocomial transmission. Unfortunately, as Dr Beccari points out most of these molecular diagnostic tools are inaccessible to the majority of physicians taking care of dialysis patients.

I believe that isolation policies for $\mathrm{HCV}$-infected dialysis patients should be developed according to the prevalence and rate of infection in a particular unit, and every effort should be undertaken to prevent spread of infection to newly admitted cases.

Bayindir Medical Center,

Cem Sungur

TR-06520 Söğütözü

Ankara,

Turkey

1. Jadoul M, Cornu C, van Ypersele de Strihou C. UCL Collaborative Study Group. Incidence and risk factors for hepatitis C seroconversion in hemodialysis: a prospective study. Kidney Int 1993; 44: 1322-1326

2. Wagner A, Kocaman A, Gessman M, Debusman E, Philipp T, Traenhart $\mathrm{O}$. Epidemiology of $\mathrm{HCV}$-infection in patients on chronic hemodialysis (abstract). J Am Soc Nephrol 1993; 4: 394A

3. Yoshida C, Vanderborght B, Stuyver L, Rouzere C, Maertens G. Tracing non-transfusional transmission of HCV infection through genomic typing of $\mathrm{HCV}$ in hemodialysis unit (abstract). In: XIIth International Congress of Nephrology, 1993, Jerusalem, 394

4. Martin P. Hepatitis C genotypes: the key to pathogenicity? Ann Intern Med 1995; 122: 227-228

\section{Complement activation by LDL-apheresis using dextran sulphate}

Sir, LDL-apheresis (LDL-aph) using dextran sulphate (DS) adsorption has been used successfully as a therapeutic tool to reduce LDL-cholesterol in familial hypercholesterolaemia [1]. Anaphylactoid reactions have been reported in patients treated with LDL-aph using DS columns [2]. These reactions have been attributed to the release of bradykinin, a potent vasodilator. However, artificial biomaterials can also activate the complement system, which may play a major role in such anaphylactoid reactions. We studied the effects of LDL-aph with DS (Kaneka LA15 Liposorber) on the complement system during three apheresis sessions, in a 25-year-old patient with familial hypercholesterolaemia.

Plasma samples were collected in EDTA before, during, and after each procedure. Complement activation products $\mathrm{C} 4 \mathrm{~d}, \mathrm{Bb}$, and SC5b-9 were measured with commercial kits (Quidel). The concentrations of several proteins, $\mathrm{CH} 50$ and AP50 were reduced after LDL-aph (see Table 1). As shown in Figure 1, complement activation products Bb and SC $5 \mathrm{~b}-9$ significantly increased at the end of LDL-aph $(\mathrm{Bb}$, in $\mu \mathrm{g} / \mathrm{ml}$, from $0.95 \pm 0.05$ to $34.4 \pm 1.1 ;$ SC $5 \mathrm{~b}-9$, in $\mathrm{ng} / \mathrm{ml}$, from $25.0 \pm 4.5$ to $830 \pm 334$ ); however, there was no significant increase in C4d. Of note, plasma samples coming out of the DS column were practically devoid of factor $\mathrm{H}$, and had increased concentrations of $\mathrm{Bb}$ and SC5b-9. There was also 
Table 1. Complement measurements after LDL-apheresis in a patient with familial hypercholesterolaemia ${ }^{a}$

\begin{tabular}{|c|c|}
\hline Protein & After LDL-apheresis ${ }^{\mathbf{b}}(\%)$ \\
\hline C3 & $69.3 \pm 1.7$ \\
\hline Factor B & $65.3 \pm 9.3$ \\
\hline D & $45.6 \pm 2.3$ \\
\hline H & $29.0 \pm 1.5$ \\
\hline I & $83.1 \pm 7.3$ \\
\hline $\mathrm{Clq}$ & $24.0 \pm 4.9$ \\
\hline $\mathrm{Clr}$ & 24.8 \\
\hline Cls & 47.7 \\
\hline C1 inhibitor & $70.3 \pm 7.7$ \\
\hline $\mathrm{C} 4$ & $78.6 \pm 3.3$ \\
\hline $\mathrm{C} 9$ & $61.0 \pm 12.1$ \\
\hline $\mathrm{CH}_{50}{ }^{\mathrm{c}}$ & $45.0 \pm 2.5$ \\
\hline $\mathrm{AP}_{50}{ }^{\mathrm{c}}$ & $41.5 \pm 0.5$ \\
\hline Total protein & $85.2 \pm 1.2$ \\
\hline Albumin & $87.0 \pm 2.3$ \\
\hline
\end{tabular}

a Antigenic concentrations, except for factor $\mathrm{D}, \mathrm{CH}_{50}, \mathrm{AP}_{50}$ (haemolytic assays). All values were in the normal range before apheresis. Total protein and albumin are used as controls.

${ }^{\circ}$ Values are expressed as the percentage of the pre-apheresis levels. Each value represents the mean $\pm S E M$ from three different apheresis sessions, except for $\mathrm{Cl} r$ and $\mathrm{Cls}$ (single values).

${ }^{\mathrm{c}}$ Standard haemolytic assays assessing classical pathway $\left(\mathrm{CH}_{50}\right)$ and alternative pathway $\left(\mathrm{AP}_{50}\right)$ of complement.

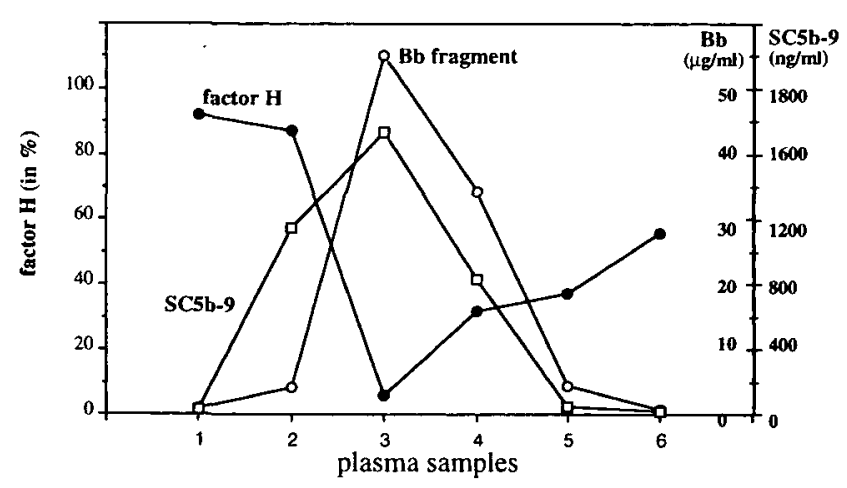

Fig. 1. Changes in the concentration of complement activation products $\mathrm{Bb}$ and SC5b-9, and of complement inhibitory protein factor $\mathrm{H}$, during LDL-apheresis with DS. Each value represents the mean from two different apheresis sessions. Plasma samples: 1. Before starting LDL-aph. 2. After separation of plasma from whole blood with filter made of polysulphone, before DS column. 3. Plasma coming out of DS column. 4. At the end of LDL-aph. 5. Two hours after the end of LDL-aph. 6. Twenty-four hours after the end of LDL-aph.

a marked $\mathrm{C} 3 \mathrm{c}$ formation (C3 conversion to $\mathrm{C} 3 \mathrm{c}$ ) as assessed by double immunodiffusion (from $<1 \%$ to $28.6 \pm 3.5 \%$, at the end of LDL-aph). Twenty-four hours after a normal LDL-aph session, no $\mathrm{C} 3 \mathrm{c}$ was detectable in plasma, and CH50, AP50 had increased to 72 and $86 \%$, respectively. In two sessions of LDL-aph, the neutrophil count (per microlitre) declined from $3875 \pm 31$ to $1645 \pm 11$ after $2 \mathrm{~h}$ of LDL-aph.

The main finding of this study was the strong activation of the alternative pathway (AP) of complement during LDLaph using DS, probably due to its deregulation in the fluid phase because of the reduction of factor $\mathrm{H}$. We could not demonstrate activation of the classical pathway. The significant reduction in factor $\mathrm{H}$ (and $\mathrm{Clq}$ ) was due to adsorption, since more than $90 \%$ of these proteins were recovered in the eluates from the DS columns. In vitro, previous work has shown that the interaction of human serum with negatively charged polystyrene sulphonate results in massive complement activation by the AP due to an immediate adsorption of factor $\mathrm{H}$ [3]. Factor $\mathrm{H}$ is an essential inhibitory protein of the AP under normal conditions; thus its adsorption or in-vivo blockade results in hypercatabolism of the AP $[3,4]$. The resulting strong complement activation by the AP may cause anaphylactoid reactions. Nafamostat mesilate, which inhibits both complement activation and the release of bradykinin, may be the anticoagulant of choice for LDL-aph using $\mathrm{DS}$, in order to avoid potential life-threatening reactions [5].

Renal Unit, Massachusetts General

M. Pascual

Hospital,

Boston, USA

E. Blanc

Hospital of Sion, Sion,

Switzerland

Department of Medicine,

Kantonsspital,

Basel, Switzerland

1. Mabuchi $\mathrm{H}$, Michishita I, Takeda $\mathrm{M}$ et al. A new low density lipoprotein apheresis system using two dextran sulfate cellulose columns in an automated column regenerating unit (LDL continuous apheresis). Atherosclerosis 1987; 68: 19-25

2. Keller C, Grutzmacher P, Bahr F et al. LDL-apheresis with dextran sulphate and anaphylactoid reactions to ACE inhibitors. Lancet 1993; 341: 60-61

3. Pascual M, Plastre O, Montdargent B, Labarre D, Schifferli JA Specific interactions of polystyrene biomaterials with factor $D$ of human complement. Biomaterials 1993; 14: 665-70

4. Veerhuis $\mathrm{R}$, Van Es LA, Daha MR. In vivo modulation of rat complement activities by infusion of anti-H antibodies. Immunobiology 1985; 170: 133-145

5. Kojima S, Harada-Shiba M, Nomura S et al. Effect of nafamostat mesilate on bradykinin generation during low-density lipoprotein apheresis using a dextran sulfate cellulose column. ASAIO Trans 1991; 37: 644-648

\section{ACE inhibitors do not decrease rHuEpo response in patients with end-stage renal failure}

Sir,

Angiotensin-converting enzyme (ACE) inhibitors have been successfully utilized to reduce haemoglobin concentrations in patients with post-transplant erythrocytosis $[1,2]$. Some authors have suggested that ACE inhibitors could decrease serum erythropoietin levels [3] and bone-marrow erythropoiesis in ESRD [4]. In this way Walter [5] has suggested that ACE inhibitors could reduce the efficacy of rHuEpo in ESRD patients. However, other studies were unable to find any effect of ACE inhibitors on haematocrit or rHuEpo dose [6].

We have studied 252 patients on haemodialysis from 17 units; 48 of them were treated with ACE inhibitors. All patients had been treated with rHuEpo for at least 6 months, and to be included in the study a patient's haemoglobin had to be over $9 \mathrm{~g} / \mathrm{dl}$ in the 3 previous months. Age, sex, months on rHuEpo treatment, months on HD, haemoglobin, haematocrit, iron metabolism, iPTH, aluminium, $\mathbf{B}_{2}$-microglobulin and $\mathrm{UI} / \mathrm{kg} /$ week of $\mathrm{rHuEpo}$ were monitored. The group treated with $A C E$ inhibitors was younger $(51 \pm 14$ versus $57 \pm 16$ years, $P<0.05$ ), but both groups were similar in 